\title{
Changing the static and dynamic lattice effects for the improvement of the ionic transport properties within the argyrodite $\mathrm{Li}_{6} \mathrm{PS}_{5-\mathrm{x}} \mathrm{Se}_{\mathrm{x}} \mathrm{I}$
}

\author{
Roman Schlem ${ }^{\mathrm{a}, \mathrm{b}}$, Michael Ghidiu, ${ }^{\mathrm{a}, \mathrm{b}}$, Sean P. Culver ${ }^{\mathrm{a}, \mathrm{b}}$, Anna-Lena Hansen ${ }^{\mathrm{c}}$, \\ Wolfgang G. Zeier*a,b \\ ${ }^{a}$ Institute of Physical Chemistry, Justus-Liebig-University Giessen, Heinrich-Buff- \\ Ring 17, D-35392 Giessen, Germany. \\ ${ }^{b}$ Center for Materials Research (LaMa), Justus-Liebig-University Giessen, \\ Heinrich-Buff-Ring 16, D-35392 Giessen, Germany. \\ ${ }^{c}$ Institute for Applied Materials - Energy Storage Systems, Karlsruhe Institute of \\ Technology, Hermann-von-Helmholtz-Platz 1, D-76344 Eggenstein- \\ Leopoldshafen, Germany
}

\begin{abstract}
The lithium argyrodites $\mathrm{Li}_{6} \mathrm{PS}_{5} \mathrm{X}(\mathrm{X}=\mathrm{Cl}, \mathrm{Br}, \mathrm{I})$ have been gaining momentum as candidates for electrolytes in all-solid-state batteries. While these materials have been well-characterized structurally, the influences of the static and dynamic lattice properties are not fully understood. Recent improvements to the ionic conductivity of $\mathrm{Li}_{6} \mathrm{PS}_{5} \mathrm{I}$ (which as a parent compound is a poor ionic conductor) via elemental substitutions have shown that a multitude of influences affect the ionic transport in the lithium argyrodites, and that even poor conductors in this class have room left for improvement.

Here we explore the influence of isoelectronic substitution of sulfur with selenium in $\mathrm{Li}_{6} \mathrm{PS}_{5-}$ ${ }_{x} \mathrm{Se}_{x} \mathrm{I}$. Using a combination of X-ray diffraction, impedance spectroscopy, Raman spectroscopy, and pulse-echo speed of sound measurements, we explore the influence of the static and dynamic lattice on the ionic transport. The substitution of $\mathrm{S}^{2-}$ with $\mathrm{Se}^{2-}$ broadens the diffusion pathways and structural bottlenecks, as well as leading to a softer more polarizable lattice, all of which lower the activation barrier and lead to an increase in the ionic conductivity. This work sheds light on ways to systematically understand and improve the functional properties of this exciting material family.
\end{abstract}

Keywords: solid electrolyte, lithium argyrodites, lattice dynamics, ionic conductivity, diffusion pathways 


\section{INTRODUCTION}

With the steadily rising demand for safer and higher-density energy storage, all-solid-state batteries have become a major research topic during the last few decades. ${ }^{1,2}$ The implementation of solid electrolytes into lithium-ion battery technologies would remove the flammability hazards of organic electrolytes, as well as offer higher mechanical stability and the potential to utilize Li metal electrodes. Nevertheless, this goal remains challenging, partially due to the search for materials with not only high ionic conductivity but that also function well within a device, having the desired morphology, compatibility with the required electrodes, and proper mechanical stability. ${ }^{3-5}$ One of the most important issues is the often low ionic conductivity of solid electrolytes, and though much progress has been made on this front, the conductivities can often span several orders of magnitude between seemingly similar (or even seemingly identical) compounds. ${ }^{6,7}$

Prominent compound classes that are being considered are the lithium conducting garnets such as $\mathrm{Li}_{7} \mathrm{La}_{3} \mathrm{Zr}_{2} \mathrm{O}_{12},{ }^{8-11}$ the $\mathrm{Li}^{+}-\mathrm{NASICON}$ 's $\mathrm{Li}_{2}{ }^{4+}\left(\mathrm{PO}_{4}\right)_{3}\left(M=\mathrm{Ti}^{4+}, \mathrm{Zr}^{4+}, \mathrm{Hf}^{4+} \text {, etc. }\right)^{12-16}$, and the lithium thiophosphates such as $\mathrm{Li}_{10} \mathrm{GeP}_{2} \mathrm{~S}_{12},{ }^{17-21} \mathrm{Li}_{2} \mathrm{~S}-\mathrm{P}_{2} \mathrm{~S}_{5}$ glasses, ${ }^{22-26}$ the thioLISICONS, ${ }^{27-31}$ and the $\mathrm{Li}_{6} \mathrm{PS}_{5} \mathrm{X}$ argyrodites. ${ }^{32-42}$ While the thiophosphates are sensitive to ambient atmosphere and may not have the largest potential window, they demonstrate high ionic conductivity and high ductility, improving the processability and resulting contact to the active materials. ${ }^{17,43,44}$

Standing out within this family of materials, the halide-containing argyrodites $\mathrm{Li}_{6} \mathrm{PS}_{5} \mathrm{X}(\mathrm{X}=$ $\mathrm{Cl}, \mathrm{Br}, \mathrm{I})$ have been explored with increasing interest. A model crystal structure of the lithium argyrodites, first reported by Deiseroth and coworkers, ${ }^{33}$ is shown in Figure 1. The unit cell consists of a face-centered cubic lattice of halide ions (Wyckoff $4 a$ ) with $\mathrm{PS}_{4}{ }^{3-}$ tetrahedra occupying the octahedral voids (P sitting on Wyckoff $4 b$ ) created by the halide ions. The two possible sites for $\mathrm{S}$ are the tetrahedral site (Wyckoff 16e) and the site Wyckoff $4 d$ that is often referred to as the "free sulfur site". ${ }^{45}$ Past studies of the lithium substructure within these argyrodites identified the existence of two possible lithium sites, where a transition state (Wyckoff $24 g$ ) for short range ion jumps between the main Wyckoff $48 h$ positions exists. ${ }^{37-}$ 39,45 It has been established that the halide ion plays a major role in the ionic transport of the argyrodites: conductivities range from $10^{-6} \mathrm{Scm}^{-1}\left(\mathrm{Li}_{6} \mathrm{PS}_{5} \mathrm{I}\right)$ to $10^{-3} \mathrm{Scm}^{-1}\left(\mathrm{Li}_{6} \mathrm{PS}_{5} \mathrm{Cl}\right)$, with $\mathrm{Li}_{6} \mathrm{PS}_{5} \mathrm{Br}$ falling in between. ${ }^{38}$ This conductivity enhancement is heavily influenced by the appearance of a site-disorder between the free sulfur anion on Wyckoff $4 d$ with the halide on $4 a ;^{37,38}$ the disorder increases from the iodide $(0 \%)$ to the chloride $(\sim 60 \%){ }^{38}$ 
A variety of different substitution series have already been performed on the argyrodites to probe underlying influences on the conductivity. ${ }^{32,36-39,45-47}$ Substitution of the halides to form solid solutions of $\mathrm{Cl} / \mathrm{Br}$ or $\mathrm{Br} / \mathrm{I}$ has demonstrated clear effects on alteration of the anion sitedisorder, with associated changes in ionic conductivity, but the disorder effects are coupled with compositional differences. ${ }^{38}$ Aliovalent substitution has also led to conductivity enhancements in the iodide structures, by substituting $\mathrm{P}$ with $\mathrm{Si}, \mathrm{Ge}$, and $\mathrm{Sn}$, with large reductions in activation energies; effects in this case are also coupled with increases in $\mathrm{Li}$ content. ${ }^{36,37}$ Introduction of $\mathrm{Se}$ in place of $\mathrm{S}$ in $\mathrm{Li}_{6} \mathrm{PS}_{5} \mathrm{Br}$ was explored as a method to increase the polarizability of the lattice, but induced competing effects by reduction of the beneficial $\mathrm{S}^{2-}$ $/ \mathrm{Br}^{-}$disorder. ${ }^{46} \mathrm{Li}_{6} \mathrm{PS}_{5} \mathrm{I}$ has the lowest ionic conductivity of the halide argyrodites (also having the lowest anion site disorder), but the capacity to improve its conductivity based on these substitutions justifies more exploration of the composition. While the aliovalent substitution described above has led to ionic conductivities in the range of $10^{-2} \mathrm{~S} \cdot \mathrm{cm}^{-1}$, it remains unclear if larger improvements may be possible using isoelectronic substitution, and by what mechanism such improvements would arise.
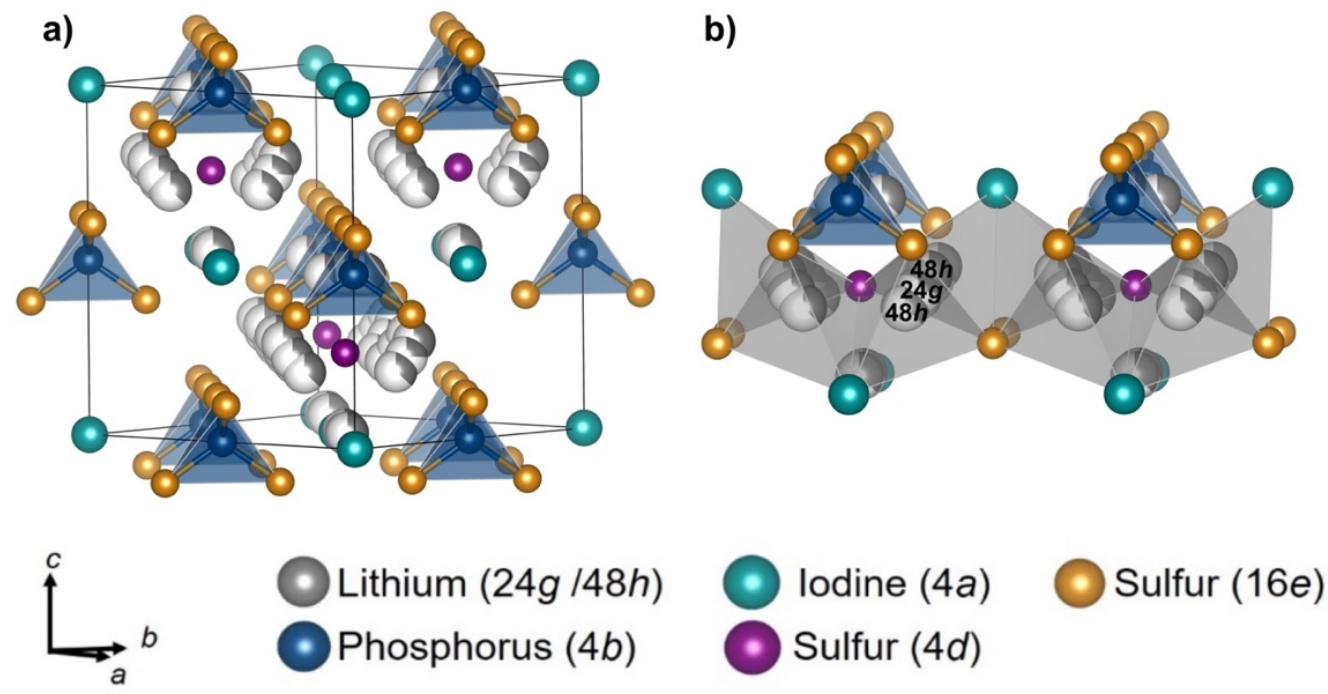

Figure 1. a) Unit cell of $\mathrm{Li}_{6} P S_{5} I$. The halide ions construct a face-centered cubic lattice with the free sulfur anion occupying half of the tetrahedral voids. Additionally, the $\mathrm{PS}_{4}{ }^{3-}$ tetrahedra occupy the octahedral voids of the face-centered cubic lattice. b) Lithium forms cluster-like arrangements around the free sulfur site. The $\mathrm{Li}^{+}$diffusion pathways are shown in grey.

Often when optimizing the ionic conductivity of solid electrolytes, effort is focused primarily on altering static structural influences such as by modifying the lithium concentration, finding materials with face-sharing polyhedra, and expanding the width of the diffusion pathways and 
structural bottlenecks. ${ }^{3,12,20,48}$ While this is a very valuable approach, recently the idea of altering the lattice dynamics through introducing softer, more polarizable anions has resurfaced. ${ }^{7,38,49-51}$ The idea of softer lattices stems from considerations of transition state theory for ionic conduction, ${ }^{52,53}$ and that a softer anion lattice due to altered anion polarizability results in weaker bonds, leading to a larger displacement amplitude for a mobile ion moving through the lattice. This larger displacement amplitude is thought to lead to faster ionic transport by lowering the associated activation barrier. ${ }^{51,54}$ This influence of the lattice dynamics on the ionic transport has recently been corroborated using the average vibrational frequency (phonon band center) as well as the Debye frequency as a descriptor for lattice dynamics. ${ }^{7,38,49-51}$ Further, recently a coupling of rotational disorder and $\mathrm{Li}^{+}$translation has been brought into the discussion in the lithium argyrodites. ${ }^{55}$

Following these ideas of lattice softening and wider diffusion pathways positively influencing ionic conduction, in this work we introduce a series of isoelectronic substitutions within $\mathrm{Li}_{6} \mathrm{PS}_{5}$ ${ }_{x} \mathrm{Se}_{x} \mathrm{I}$. Using a combination of structural analysis via Rietveld refinements against X-ray diffraction data and impedance spectroscopy to assess ionic transport, we show the influences of selenium substitution in $\mathrm{Li}_{6} \mathrm{PS}_{5-x} \mathrm{Se}_{x} \mathrm{I}$. Furthermore, by measuring the Raman vibrational spectra and pulse-echo speed of sound to obtain the changing Debye frequencies, the changing lattice dynamics are explored. The substitution of $\mathrm{S}$ with Se leads to an increase of the ionic conductivity of up two orders of magnitude from the unsubstituted $\mathrm{Li}_{6} \mathrm{PS}_{5} \mathrm{I}$. This increase in conductivity stems from a linear decrease of the activation barrier that can be explained by the changing static structural features of wider diffusion pathways as well as a softer more polarizable lattice.

\section{EXPERIMENTAL METHODS}

Synthesis. The preparations for all samples within the $\mathrm{Li}_{6} \mathrm{PS}_{5-x} \mathrm{Se}_{x} \mathrm{I}$ substitution series were carried out under argon atmosphere. $\mathrm{Li}_{2} \mathrm{~S}$ (Alfa Aesar, $99.9 \%$ ), $\mathrm{P}_{2} \mathrm{~S}_{5}$ (Sigma Aldrich, 99 \%) and Se (Alfa Aesar, $99.999 \%$ ) were used as received. LiI (Alfa Aesar, $99.95 \%$ ) was dried for at least $48 \mathrm{~h}$ at $450{ }^{\circ} \mathrm{C}$ under dynamic vacuum to remove the majority of $\mathrm{LiI} \cdot\left(\mathrm{H}_{2} \mathrm{O}\right.$ ) (see Supporting Information Figure $\mathrm{S} 1)$. $\mathrm{Li}_{2} \mathrm{Se}$ was prepared by a gas phase reaction of Se with lithium metal (Alfa Aesar, $99.9 \%$ ), using the same approach as recently reported. ${ }^{49}$ The chemicals were mixed in their stoichiometric ratio, hand ground in an agate mortar for at least 15 minutes and pressed into dense pellets before loading into $\sim 10 \mathrm{~cm}$ long quartz ampoules (10 $\mathrm{mm}$ inner diameter) that had each been pre-heated at $800^{\circ} \mathrm{C}$ for at least 1.5 hours under dynamic vacuum to ensure the removal of all traces of water. The ampoules containing the powder 
mixtures were sealed under vacuum before the reactions were performed at $550{ }^{\circ} \mathrm{C}$ for 2 weeks and afterwards air quenched to room temperature. Quenching of the ampoules was needed for a selenium content of $x>3$, in order to prevent phase segregation, therefore, all samples were quenched to ensure better comparability.

X-Ray diffraction and structural analysis. X-Ray diffraction experiments were carried out on a STOE Stadi P in Debye-Scherrer geometry. The diffractometer is equipped with two DECTRIS MYTHEN detectors and a silver anode $(\lambda=0.5594 \AA, \mathrm{Ge}(111)$ monochromator) Rietveld refinements were carried out using the TOPAS software package. ${ }^{56} \mathrm{~A}$ visual assessment, as well as the fit indicator goodness-of-fit (GOF) were used to determine the quality of the fits. A Chebyshev function of 10 parameters was used to fit the background. For the peak shape modelling a modified Thomson-Cox-Hastings pseudo-Voigt function was employed. The used constraints can be found in the structural tables in the Supporting Information.

Electrochemical impedance spectroscopy. Electrical conductivities were measured using AC impedance spectroscopy, using pellets with a thickness of $\sim 2 \mathrm{~mm}$ and a geometric density of $>85 \%$ packed into argon filled pouch cells. The pellets were isostatically pressed and afterwards annealed for 15 minutes at $550{ }^{\circ} \mathrm{C}$. Evaporated gold electrodes $\left(0.53 \mathrm{~cm}^{2}\right)$ were used for contacting. The electrochemical impedance analysis (EIS) was conducted in the temperature range of $-40{ }^{\circ} \mathrm{C}$ to $60{ }^{\circ} \mathrm{C}$ using a VMP300 impedance analyzer (Biologic) at a frequency range of $7 \mathrm{MHz}$ to $100 \mathrm{mHz}$ with an amplitude of $10 \mathrm{mV}$. In order to obtain statistical information, three pellets of each composition were prepared and measured separately.

Speed of sound measurements. Pulse-echo speed of sound measurements were performed on consolidated discs using an Epoch 600 (Olympus) with 5 MHz transducers for longitudinal and transverse speeds of sound. The samples were coated with a thin layer $(<200 \mathrm{~nm})$ of gold in order to prevent side reactions of the sample with the couplant honey. All measurements were performed under nitrogen atmosphere. Three pellets of each composition were measured, with at least two responses per pellet, and these three data sets used to determine the average speeds of sound, thereby reducing the measurement uncertainty.

Raman spectroscopy. The samples were placed on a microscopy glass slide and sealed airtight with grease and a glass coverslip. A Senterra Raman spectrometer (Bruker Optics) was used with an excitation wavelength of $532 \mathrm{~nm}$. A resolution of $3-5 \mathrm{~cm}^{-1}$ was achieved in the 
range of $47-1548 \mathrm{~cm}^{-1}$ with a $20 \mathrm{x}$ magnification lens using an integration time of $3 \mathrm{~s}$ per spectrum and 100 spectra per measurement. A low laser power of $0.2 \mathrm{~mW}$ was used to prevent sample decomposition.

\section{RESULTS AND DISCUSSSION}

Structural changes. The solid solutions of $\mathrm{Li}_{6} \mathrm{PS}_{5-x} \mathrm{Se}_{x} \mathrm{I}$ were chosen in order to observe lattice effects independent of any halide anion site-disorder (due to the negligible disorder present in the I argyrodites), ${ }^{37,38,46}$ as this would further complicate the structure-transport analysis, with the benefit that the compositions studied are distinguishable due to differences in X-ray scattering form factors of S, Se and I.
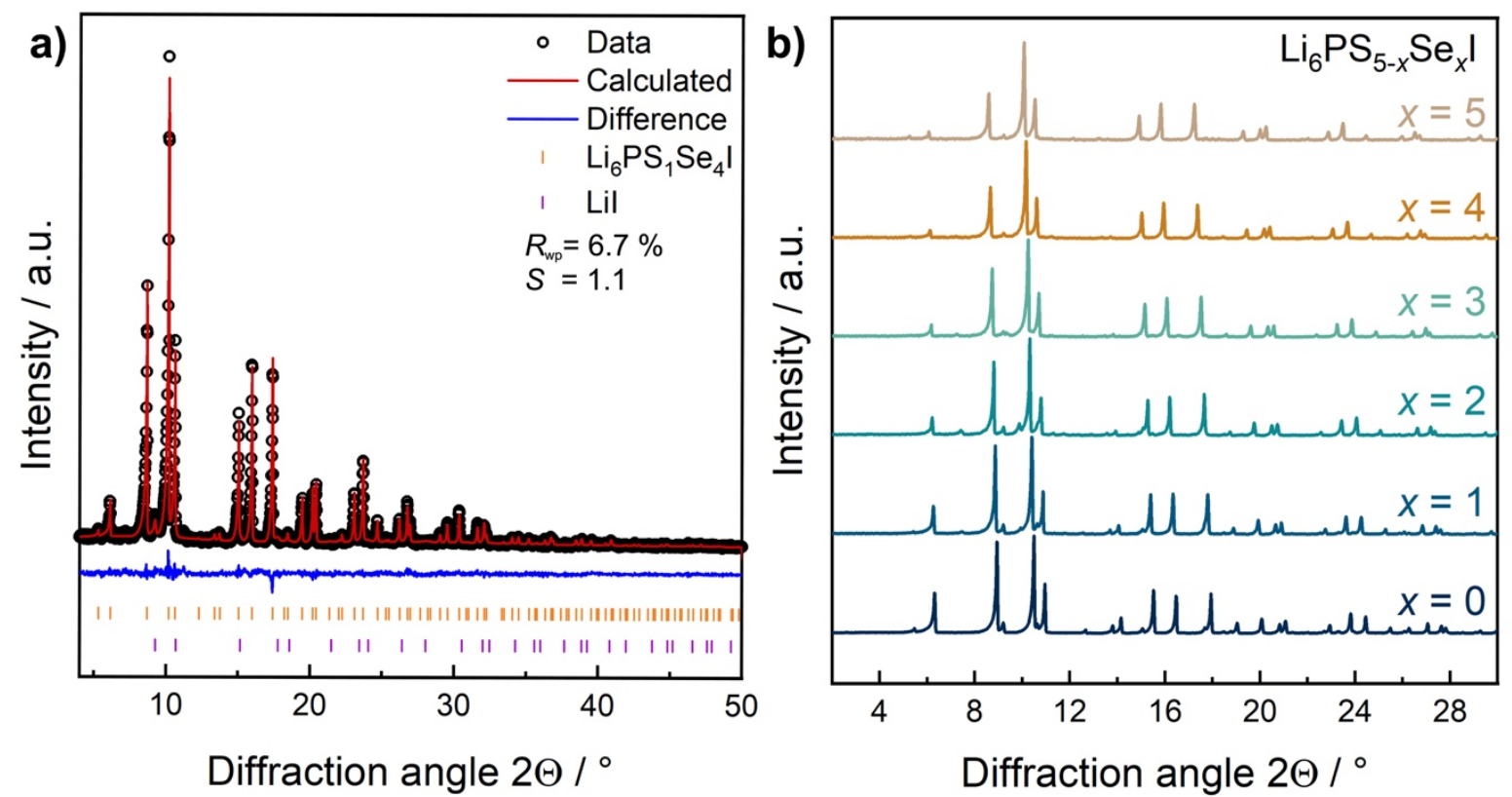

Figure 2. a) Rietveld-refinement example of $\mathrm{Li}_{6} \mathrm{PS}_{1} \mathrm{Se}_{4} \mathrm{I}$ including the LiI impurity phase ( 3.5 wt.\%) and b) all obtained diffraction patterns of the solid solutions $\mathrm{Li}_{6} P S_{5-x} S e_{x} I$.

In order to study the structural changes within the $\mathrm{Li}_{6} \mathrm{PS}_{5-x} \mathrm{Se}_{x} \mathrm{I}$ solid solution series, compositions with a step size of $\mathrm{x}=1$ of increasing selenium content were synthesized by classical methods and investigated using Rietveld refinements against X-ray diffraction data. Figure 2 shows a representative Rietveld refinement for the composition of $\mathrm{Li}_{6} \mathrm{PS}_{1} \mathrm{Se}_{4} \mathrm{I}$ and all X-ray diffraction patterns for comparison. All other refinement results can be found in the Supporting Information (Figures S2 - S7). While a minor contribution of LiI can be found as an impurity phase, a linear expansion of the lattice parameters caused by the larger ionic radius of selenium $(198 \mathrm{pm})$ in comparison to sulfur $(184 \mathrm{pm})^{57}$ can be observed, thus obeying 
Vegard's rule ${ }^{58}$ (Figure 3a). The absence of a major selenium rich impurity phase indicates a true solid solution for the $\mathrm{Li}_{6} \mathrm{PS}_{5-\mathrm{x}} \mathrm{Se}_{\mathrm{x}} \mathrm{I}$ system without the existence of a solubility limit. Small impurity fractions of $\operatorname{LiI}\left(\mathrm{H}_{2} \mathrm{O}\right)$ can still be found for the solid solutions of $\mathrm{x}<3$, which seem to only occur when $\mathrm{Li}_{2} \mathrm{~S}$ is used as a precursor. Additional structural features such as the $\mathrm{Se}$ occupancy on the free chalcogenide site (Wyckoff $4 d$ ), the Se occupancy on the $\mathrm{PS}_{4-\mathrm{x}} \mathrm{Se}_{\mathrm{x}}{ }^{3-}$ tetrahedra (Wyckoff 16e) and the volume of the $\mathrm{P}(\mathrm{S} / \mathrm{Se}) 4^{3-}$ tetrahedra can be found in Figure 3 as well. Similar to the lattice parameters, the incorporation of selenium leads to an expansion of the $\mathrm{P}(\mathrm{S} / \mathrm{Se}) 4^{3-}$ tetrahedra. With increasing degree of substitution, the amount of sulfur and selenium on the free sulfur site as well as on the $\mathrm{P}(\mathrm{S} / \mathrm{Se}) 4^{3-}$ tetrahedra increases (Figure $3 \mathrm{c}$ and 3d). The overall change in the Se occupancy on both lattice sites suggests an even distribution between the sites and no clear site preference for Se. While unsubstituted $\mathrm{Li}_{6} \mathrm{PS}_{5} \mathrm{I}$ exhibits no significant site-disorder between $\mathrm{I}^{-}$and $\mathrm{S}^{2-}$, a minor change in the disorder can be found during Se substitution, to a maximum of $\sim 7 \%$ (see Supporting Information Figure S8). ${ }^{38}$
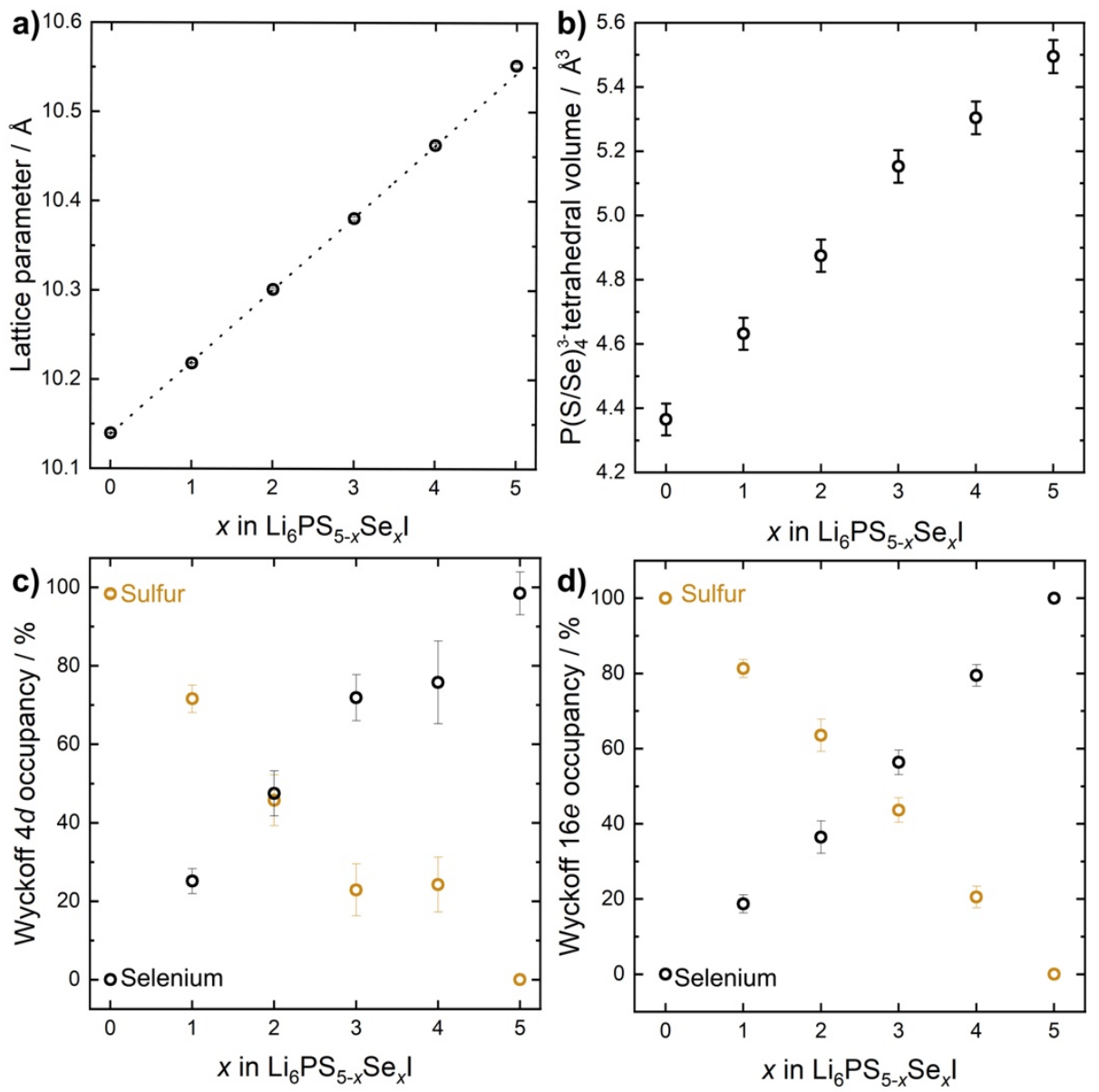

Figure 3. a) The incorporation of the larger selenium leads to an expansion of the unit cell, as well as the b) $P(\mathrm{~S} / \mathrm{Se})_{4}^{3-}$ tetrahedral volume. A true solid solution is indicated by the linear expansion of the lattice, indicating the absence of a solubility limit for selenium within $\mathrm{Li}_{6} P S_{5-}$ 
${ }_{x} S e_{x} I$. c) Partial chalcogenide occupancies on the free sulfur site (Wyckoff $4 d$ ) and the d) tetrahedral site (Wyckoff 16e).

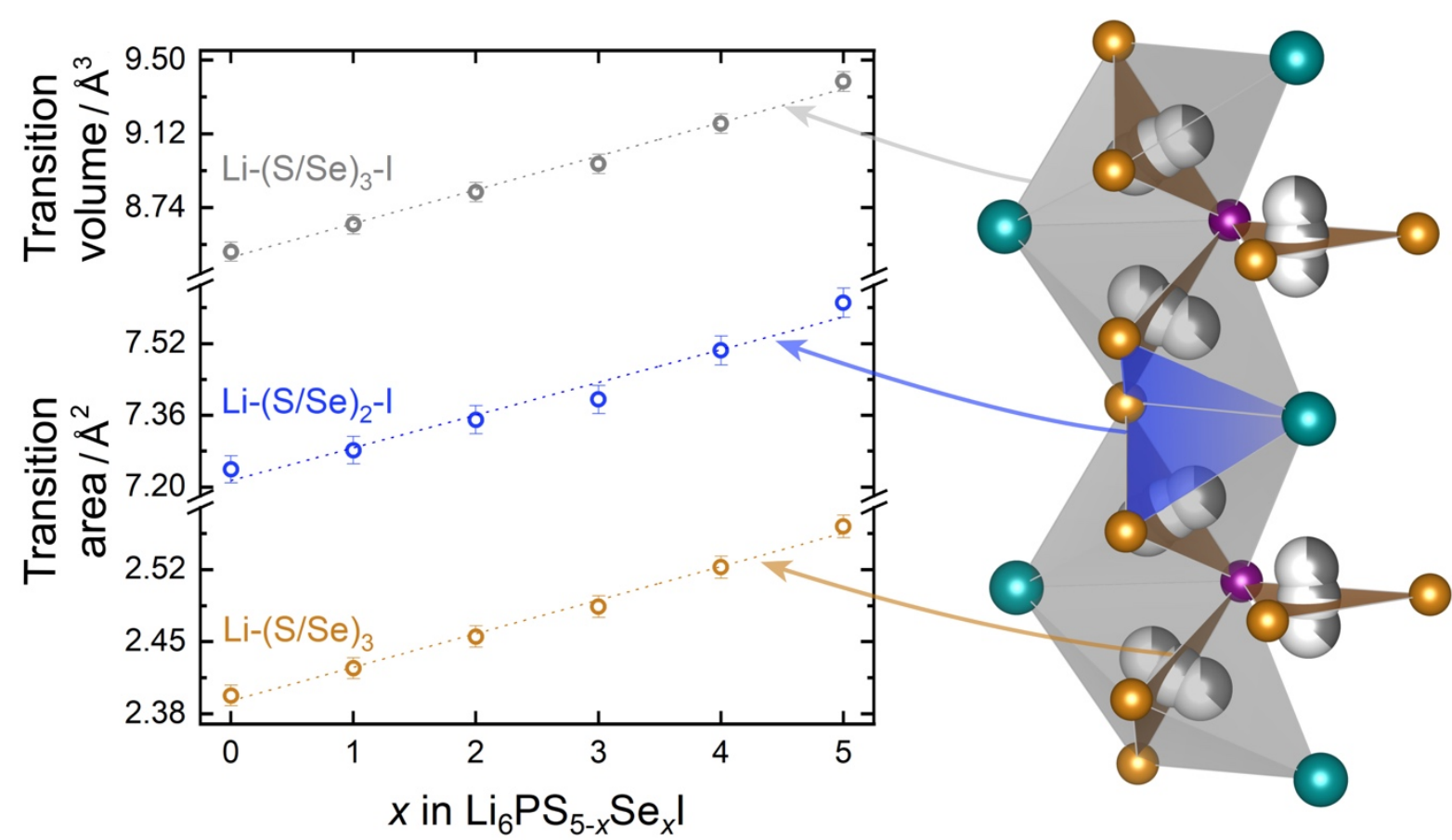

Figure 4. A steadily increasing selenium occupancy and expanding lattice leads to wider diffusion pathways for $\mathrm{Li}^{+}$. Shown are the resulting $\mathrm{Li}^{+}$coordination-tetrahedra $\left(\mathrm{Li}-(\mathrm{S} / \mathrm{Se})_{3}-\mathrm{I}\right)$ in grey, the long-range inter-cage jump transition area $\left(\mathrm{Li}-(\mathrm{S} / \mathrm{Se})_{2} \mathrm{I}\right)($ blue) and the short-range intra-cage jump transition area $\left(\mathrm{Li}(\mathrm{S} / \mathrm{Se})_{3}\right)$ (orange).

The expansion of the $\mathrm{P}(\mathrm{S} / \mathrm{Se}) 4^{3-}$ tetrahedra is in close structural vicinity to $\mathrm{Li}^{+}$and hence an influence on the diffusion pathways can be expected. Two different $\mathrm{Li}^{+}$positions are known in $\mathrm{Li}_{6} \mathrm{PS}_{5} \mathrm{X}$, namely the Wyckoff $48 h$ and $24 g$, which form cages around the free $\mathrm{S}^{2-}$ in the tetrahedral voids of the face-centered cubic lattice. ${ }^{33,45}$ These two positions give rise to three different jump processes: (i) a so-called doublet jump from $48 h$ via $24 g$ to another $48 h$ position, (ii) an intra cage-jump of $48 h$ to $48 h$, and (iii) an inter-cage jump from position $48 h$ to $48 h$. While the inter-cage jumps seem to be the rate-limiting step for the $\mathrm{Li}^{+}$jump process and overall measurable ionic conductivity, ${ }^{37,38,45,59}$ the structural features around all jumps are of interest as they directly influence the diffusion behavior for lithium.

Figure 4 shows the evolution of the $\mathrm{Li}-(\mathrm{S} / \mathrm{Se})_{3}$ triangular transition area and the corresponding $\mathrm{Li}-(\mathrm{S} / \mathrm{Se})_{3} \mathrm{I}$ tetrahedra with increasing Se content, both of which describe the pathways of the doublet jump and intra-cage jump. Further shown is the side of a $\mathrm{Li}-(\mathrm{S} / \mathrm{Se})_{3} \mathrm{I}$ tetrahedron, coordinated by $\mathrm{I},(\mathrm{S} / \mathrm{Se})$ and $(\mathrm{S} / \mathrm{Se})$. This side of the tetrahedron is the bottleneck for the inter- 
cage jump as the lithium ions will have to pass through this triangle during the jump from one cage to another. While the X-ray diffraction data makes it impossible to locate the $\mathrm{Li}^{+}$in the structure, Figure 3 shows that all anionic structural features describing the diffusion pathways widen geometrically, which will likely affect the overall ionic transport.

Lattice dynamics. Recently, there has been increased interest in understanding the influence of the lattice vibrations and dynamics on the ionic transport in ionic conductors. ${ }^{38,50,60} \mathrm{With}$ the incorporation of a more polarizable anion, the lattice vibrational properties were observed to soften, which in turn affects the ionic transport by decreasing the enthalpy and entropy of migration. ${ }^{38,49-51}$ Further, it was recently shown that additional polyhedral anion dynamics exist that are coupled to the $\mathrm{Li}^{+}$translation. ${ }^{55}$ However, in the $\mathrm{Li}_{6} \mathrm{PS}_{5} \mathrm{I}$ series reported here, the $\mathrm{Li}^{+}$ ion jumps and rotational dynamics are independent of each other, which beneficially prevents further complication of the analysis. In this work, the resulting lattice dynamics are assessed using the optical and acoustic modes of the dispersion relation. ${ }^{61,62}$ Raman spectroscopy and the measurement of the speed of sound within a material can give reliable information regarding the phonon dynamics and average phonon frequencies. ${ }^{38,49,50,63-65}$

Figure 5a shows the influence of increasing selenium occupancy on the stretching mode of the $\mathrm{PS}_{4-\mathrm{y}} \mathrm{Se}_{\mathrm{y}}{ }^{3-}$ tetrahedra. First, the appearance of multiple, isolated $\mathrm{PS}_{4-\mathrm{y}} \mathrm{Se}_{\mathrm{y}}{ }^{3-}$ tetrahedral species can be observed for the mixed $\mathrm{S} / \mathrm{Se}$ compounds that correspond to a symmetry reduction from $T_{\mathrm{d}}$ to $C_{3 \mathrm{v}}$ and eventually $C_{2 \mathrm{v}}$ with increasing degree of substitution. Such a behavior has already been reported for the $\mathrm{Na}_{3} \mathrm{PS}_{4-x} \mathrm{Se}_{x}$ system by Krauskopf et al. ${ }^{50}$, who also showed that a binomial distribution well describes the occurrence of such mixed tetrahedra. This interchangeability of sulfur and selenium on the tetrahedral sites suggests similar formation energies for ordered and partially disordered tetrahedra even for different types of compounds. Figure $5 \mathrm{~b}$ shows the correlation between the average crystallographic bond length (and therefore bond strength) and the corresponding Raman shift. With increasing Se content and increasing bond length, the Raman shift decreases. The $\mathrm{PSe}_{4}{ }^{3-}$ tetrahedra show an increasing Raman shift at higher Se content which trends counter to the overall observed changes, suggesting some additional underlying local processes. Overall, the decreasing Raman shift with respect to the change of the average bond length indicates a softening of the optical phonons when substituting $\mathrm{S}$ for $\mathrm{Se}$ in $\mathrm{Li}_{6} \mathrm{PS}_{5-x} \mathrm{Se}_{x} \mathrm{I}$, similar to the one recently found for $\mathrm{Na}_{3} \mathrm{PS}_{4-x} \mathrm{Se}_{x}{ }^{50}$ 

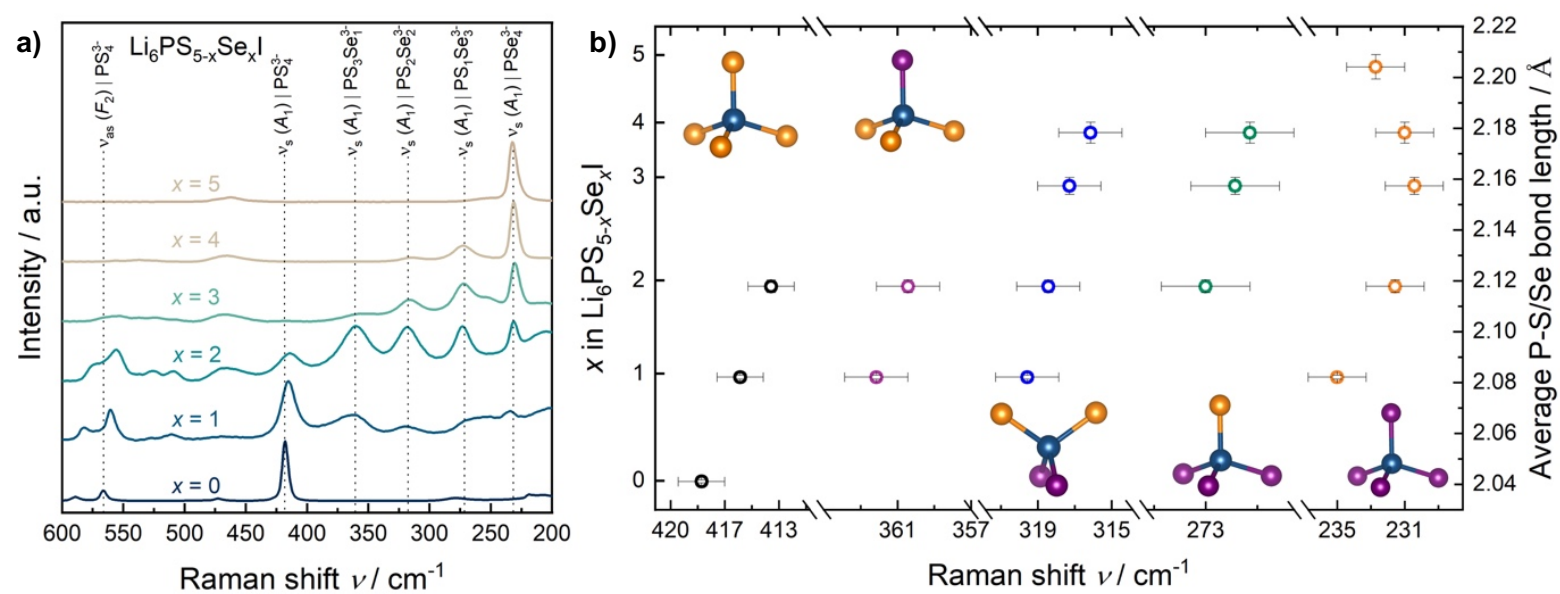

Figure 5. a) Obtained Raman spectra showing the formation of new, isolated $P S_{4-y} S e_{y}$ tetrahedra. b) The vibrational energy reduction within the different $P S_{4-y} S e_{y}$ tetrahedra with respect to the average bond length indicates a softening of the optical vibrational modes.

As the Raman measurements only give insight into the optical phonons, the acoustic phonons were assessed using speed of sound measurements. Measuring the longitudinal and transverse speeds of sound probes the group velocity of lattice oscillations close to the center of the Brillouin zone, which can be expressed as the slope of the dispersion relation in this region (see inset Figure $6 \mathrm{~b}) .{ }^{50}$ Using a calculated mean speed of sound $v_{\mathrm{m}}$ and average volume per atom $V$, the Debye frequency $v_{D}$ can be obtained and it can be used as a descriptor of the average lattice dynamics. ${ }^{50,65}$

$$
v_{D}=\left(\frac{3}{4 \pi V}\right)^{\frac{1}{3}} v_{\mathrm{m}}, \text { with } v_{\mathrm{m}}=\left(\frac{3}{v_{\text {long }}^{-3}+2 v_{\text {trans }}^{-3}}\right)^{\frac{1}{3}}
$$

Figure 6 shows the different speeds of sound and the resulting Debye frequencies of the lattices of these solid solutions. As expected, with increasing selenium content in $\mathrm{Li}_{6} \mathrm{PS}_{5-x} \mathrm{Se}_{x} \mathrm{I}$ the speed of sound and Debye frequencies decrease, indicating an overall softening of the lattice. In other words, the larger and more polarizable Se anion leads to a decrease in the energy of the lattice vibrations and softer/weaker bonding in the framework. 

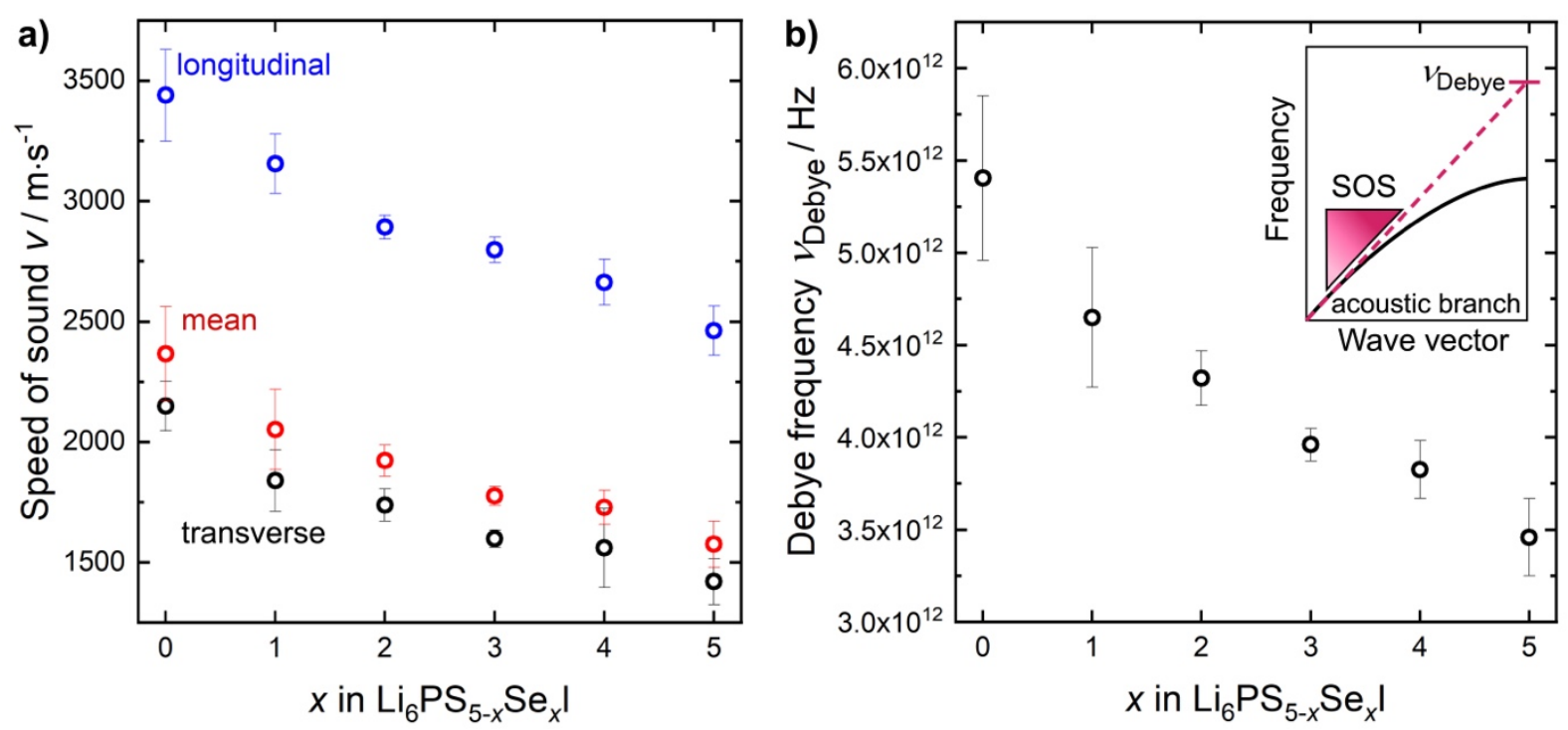

Figure 6. a) Observed speeds of sound and b) Debye frequencies indicate a softening of the acoustic phonons with increasing Se content in $\mathrm{Li}_{6} P S_{5-x} \mathrm{Se}_{x} I$. Error bars correspond to standard deviations obtained from measurements on three samples per composition. The inset shows a schematic relation of the Debye frequency to the speed of sound within a phonon dispersion curve.

Ionic transport. The ionic transport properties of the $\mathrm{Li}_{6} \mathrm{PS}_{5-x} \mathrm{Se}_{x} \mathrm{I}$ series were assessed using temperature-dependent impedance spectroscopy. Representative room-temperature Nyquistplots, as well as the resulting Arrhenius plots, are shown in Figure 7. Small grain boundary contributions and an electrode roughness can be seen that lead to a slight asymmetry for the low frequency region of the semicircle. The conductivities shown here represent the bulk contribution of ionic transport as all processes can be distinguished based on the process capacitances. ${ }^{66-68}$ For instance in $\mathrm{Li}_{6} \mathrm{PSe}_{5} \mathrm{I}$, the bulk contributions show capacitances in the range of $59 \mathrm{pF} \cdot \mathrm{cm}^{-2}$ and the grain boundary/electrode roughness contributions in the range of $34 \mathrm{nF} \cdot \mathrm{cm}^{-2}$. Further, alpha factors of up to 0.96 for the bulk contribution indicate the ideality of the process. ${ }^{69}$

Figure 7 shows the obtained Nyquist plots of all compositions, normalized to the pellet thickness for comparison. The employed equivalent circuit is shown in the inset. The activation barriers for ionic migration and the corresponding conductivities, both extracted from the Arrhenius plots, can also be found. A significant, linear decrease of the activation barrier can be observed with increasing Se content. This decrease of the activation barrier is governed by an increase of ionic conductivity of up to 2 orders of magnitude leading to an ionic conductivity of $0.28 \mathrm{mS} \cdot \mathrm{cm}^{-1}$ compared to $0.0025 \mathrm{mS} \cdot \mathrm{cm}^{-1}$ for the unsubstituted $\mathrm{Li}_{6} \mathrm{PS}_{5} \mathrm{I}$. 


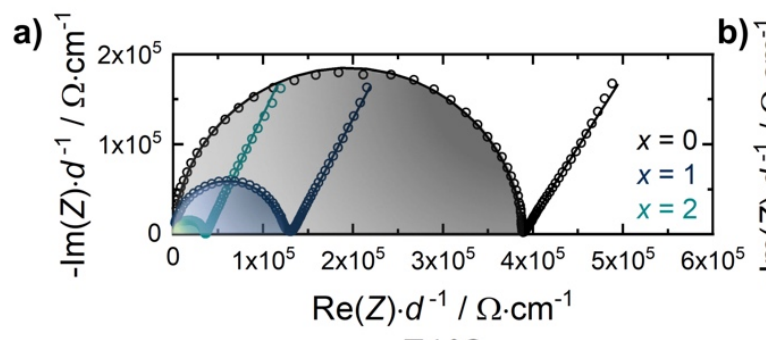

$T /{ }^{\circ} \mathrm{C}$

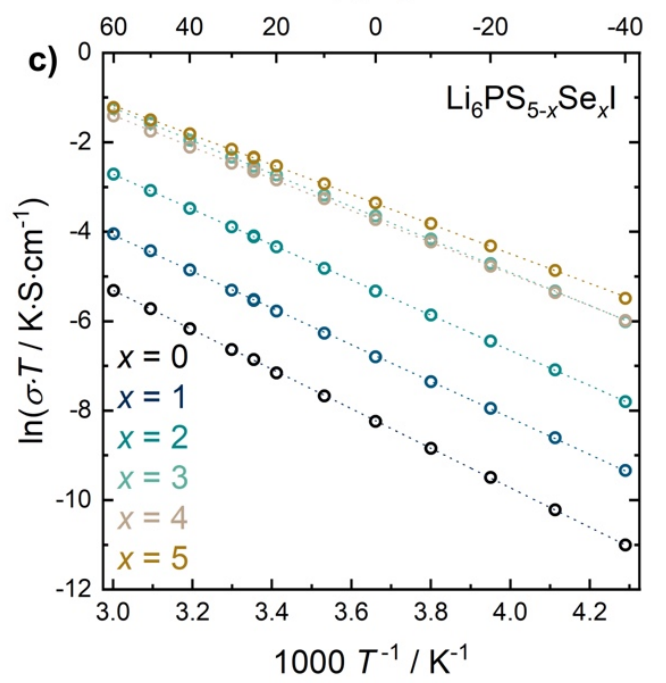

b) ${ }^{\top} \varepsilon$
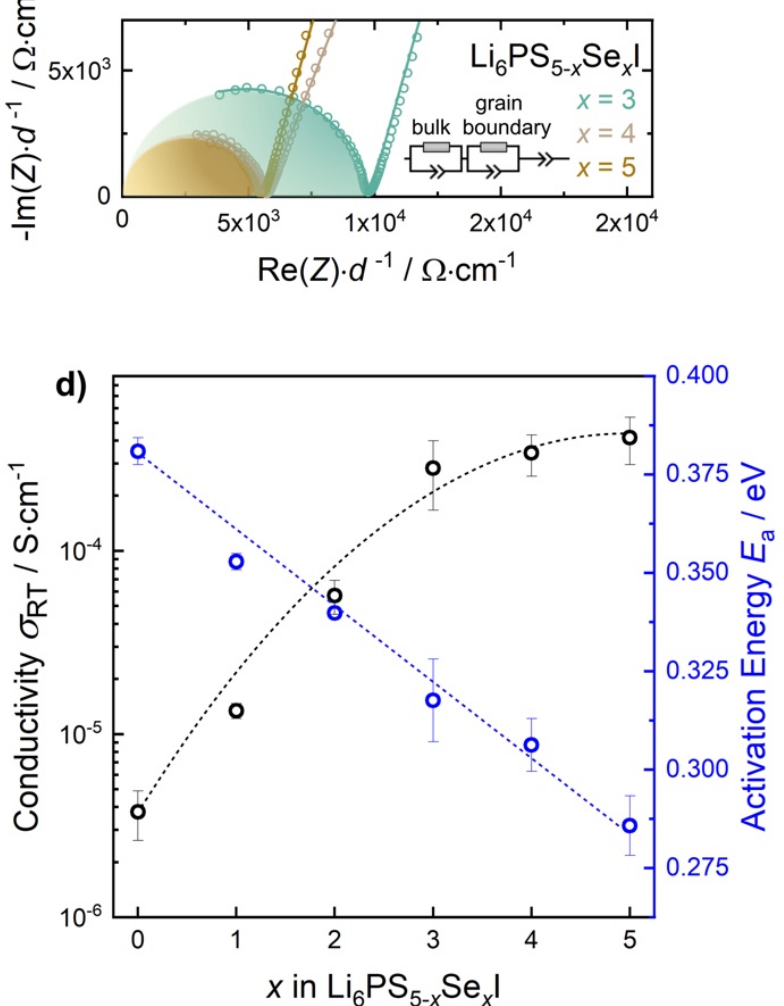

Figure 7: a) and b) Impedance spectra of all compositions of $L i_{6} P S_{5-x} S e_{x} I$ at room temperature, showing the ideality of the measurements and the equivalent circuit that was used for fitting. $c$ ) Calculated Arrhenius diagrams showing the good fit quality and d) room-temperature conductivity and activation barriers. The Se substitution leads to a decreasing activation barrier and results in an increased conductivity over multiple orders of magnitude. Error bars correspond to standard deviations obtained from measurements on three samples per composition.

Structure - property relationships. Figure 8 shows the influence of the expanding lithium transition polyhedra on the measured activation barrier as well as the correlation to the lattice dynamics. Using the three different structural descriptors for the diffusion pathway introduced above, i.e. (i) the triangular transition areas for the short range doublet jump and (ii) the long range intercage jump as well as the (iii) resulting $\mathrm{Li}(\mathrm{S} / \mathrm{Se})_{3} \mathrm{I}$ tetrahedral volume, a clear correlation can be found. With increasing width of the diffusion pathways that govern the jumps within the $\mathrm{Li}^{+}$cage and from cage to cage (inter-cage jump), the activation barrier decreases (Figure 8a). As lithium jumps between adjacent sites, the ions surrounding the diffusion pathway have to be displaced. This is often referred to as the bottleneck for diffusion. By 
increasing the static structure, and with it the size of this bottleneck, the required energetic barrier for the ionic jump is reduced.

In addition to the width of the diffusion pathways, the overall softness and polarizability of the lattice also plays a role (Figure $8 \mathrm{~b}$ ). With increasing selenium content, the lattice softens and less energy needs to be expended displacing the lattice during the jump. This influence of an increasing lattice polarizability leading to a reduced activation barrier has recently been shown in a variety of $\mathrm{Li}^{+}$and $\mathrm{Na}^{+}$superionic conductors. ${ }^{38,49,50}$ In this work, the minor disorder induced on the anion site (Figure S8) does not seem to correlate to the boost in conductivity, suggesting the lattice softening and diffusion pathway widening as a primary mechanism for better ionic transport. This is in contrast to work on $\mathrm{Li}_{6} \mathrm{PS}_{5} \mathrm{Br}$, where Se subsitution induced a substantial decrease in anion site disorder, convoluting the effects on conductivity. ${ }^{46}$

Overall, $\mathrm{Li}_{6} \mathrm{PS}_{5-\mathrm{x}} \mathrm{Se}_{\mathrm{x}} \mathrm{I}$ clearly represents an example in which lattice softening coupled with diffusion pathway widening strongly influences ionic transport, and shows great promise if it can be engineered without affecting beneficial anion disorder in the other argyrodites. 

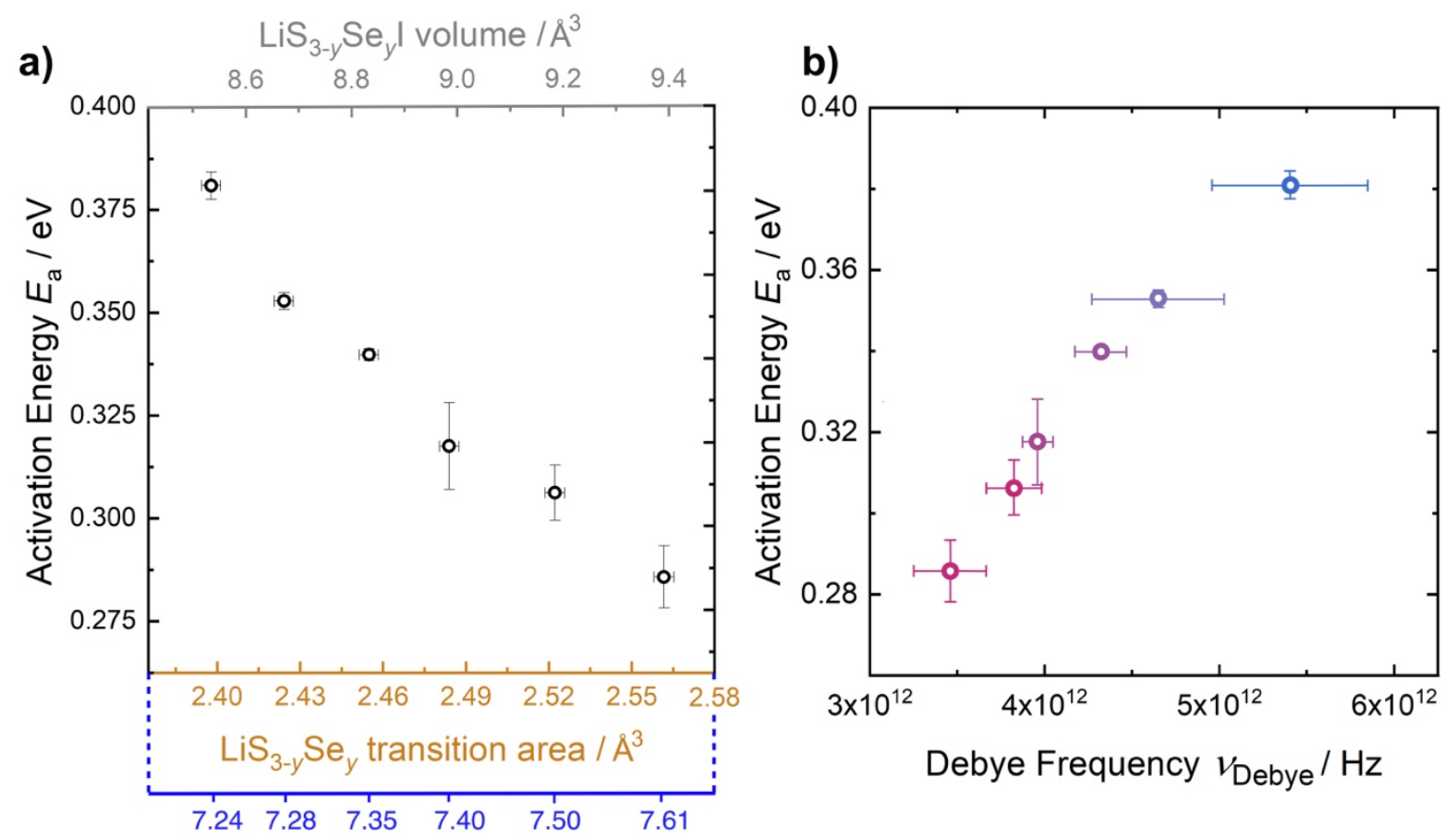

c)
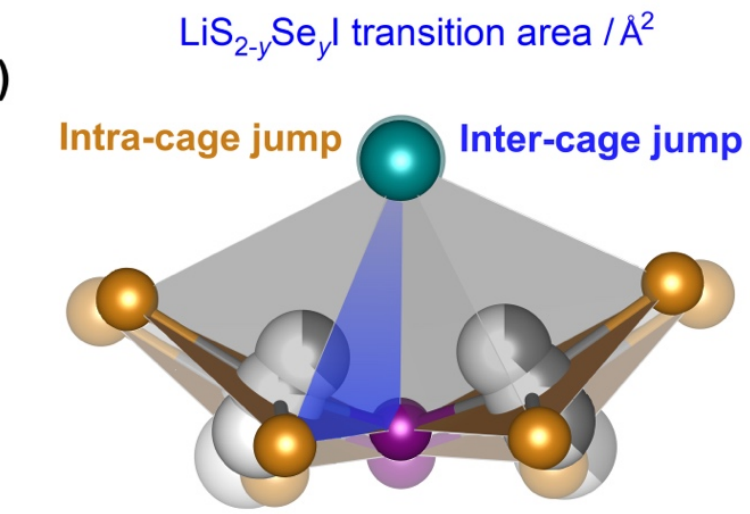

static effect

d)

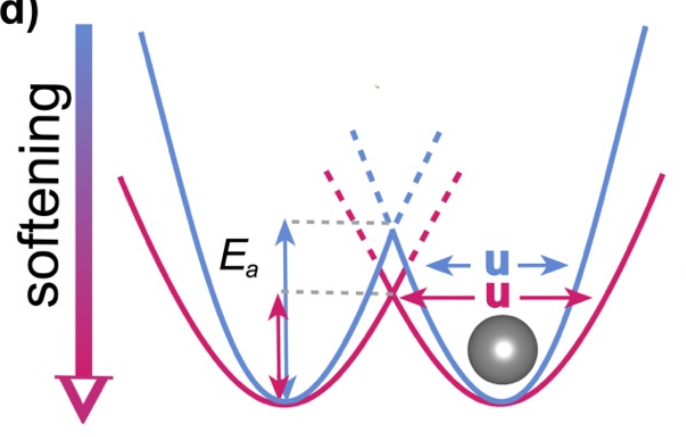

dynamic effect

Figure 8. a) Structure property relationship between the activation barrier and the transition area for Lithium diffusion. The decreasing activation barrier is attributed to a simplified transition with increasing area size. b) Furthermore, a direct correlation between the lattice dynamics and the activation barrier is shown. The Debye frequency shows a linear relation to the activation barrier. c) Schematic on the diffusion pathways and influence of wider bottlenecks, related to the data in a). d) Schematic of the effect of lattice softening on the ionic jump. The changing Debye frequency and softer lattice leads to wider oscillator potentials and lower barriers, related to the data in (b). 


\section{CONCLUSION}

In this work, we report the successful incorporation of Se into the argyrodite structure leading to the substitution series of $\mathrm{Li}_{6} \mathrm{PS}_{5-\mathrm{x}} \mathrm{Se}_{\mathrm{x}} \mathrm{I}$. The linear lattice expansion and absence of a selenium rich impurity phase indicate the formation of true solid solutions. The incorporation of the larger and more polarizable anion results in a decrease in the observable activation barrier for ionic migration, resulting in a conductivity increase of around 2 orders of magnitude. The decreasing activation barrier can be directly related to changes in the static lattice structure and its lattice dynamics, showing that wider diffusion pathways and more polarizable lattices can positively influence ionic conduction in the argyrodite superionic conductors. Moreover, given that the conductivity of the iodide argyrodite was drastically increased without the influence of increased anion site disorder (which has been suggested to play a major role in the series of the halide argyrodites), this work highlights the effects of lattice softening that could be exploited for improving other known compositions.

\section{Supporting Information.}

The effect of pre-drying of the LiI precursor is shown. In addition, all structural data as obtained from Rietveld refinements can be found here.

\section{AUTHOR INFORMATION}

\section{Corresponding Authors}

*wolfgang.g.zeier@pc.jlug.de;

\section{Notes}

The authors declare no competing financial interests.

\section{Acknowledgements}

The research was supported by the Federal Ministry of Education and Research (BMBF) within the project FESTBATT under grant number 03XP0177A and 03XP0176A. S.C. gratefully acknowledges the Alexander von Humboldt Foundation for financial support through a Postdoctoral Fellowship. 


\section{References}

(1) Kato, Y.; Hori, S.; Saito, T.; Suzuki, K.; Hirayama, M.; Mitsui, A.; Yonemura, M.; Iba, H.; Kanno, R. High-Power All-Solid-State Batteries Using Sulfide Superionic Conductors. Nat. Energy 2016, 1, 16030.

(2) Janek, J.; Zeier, W. G. A Solid Future for Battery Development. Nat. Energy 2016, 1,pp. 16141.

(3) Culver, S. P.; Koerver, R.; Krauskopf, T.; Zeier, W. G. Designing Ionic Conductors: The Interplay between Structural Phenomena and Interfaces in Thiophosphate-Based SolidState Batteries. Chem. Mater. 2018, 30, 4179-4192.

(4) Ghidiu, M.; Ruhl, J.; Culver, S. P.; Zeier, W. G. Solution-Based Synthesis of Lithium Thiophosphate Superionic Conductors for Solid-State Batteries: A Chemistry Perspective. J. Mater. Chem. A 2019, 7, 17735-17753.

(5) Kato, Y.; Shiotani, S.; Morita, K.; Suzuki, K.; Hirayama, M.; Kanno, R. All-Solid-State Batteries with Thick Electrode Configurations. J. Phys. Chem. Lett. 2018, 9, 607-613.

(6) Asano, T.; Sakai, A.; Ouchi, S.; Sakaida, M.; Miyazaki, A.; Hasegawa, S. Solid Halide Electrolytes with High Lithium-Ion Conductivity for Application in 4 V Class BulkType All-Solid-State Batteries. Adv. Mater. 2018, 30, 1803075.

(7) Muy, S.; Voss, J.; Schlem, R.; Koerver, R.; Sedlmaier, S. J.; Maglia, F.; Lamp, P.; Zeier, W. G.; Shao-Horn, Y. High-Throughput Screening of Solid-State Li-Ion Conductors Using Lattice-Dynamics Descriptors. iScience 2019, 16, 270-282.

(8) Cussen, E. J. Structure and Ionic Conductivity in Lithium Garnets. J. Mater. Chem. 2010, 20, 5167-5173.

(9) Li, Y.; Han, J. T.; Wang, C. A.; Vogel, S. C.; Xie, H.; Xu, M.; Goodenough, J. B. Ionic Distribution and Conductivity in Lithium Garnet $\mathrm{Li}_{7} \mathrm{La}_{3} \mathrm{Zr}_{2} \mathrm{O}_{12}$. J. Power Sources 2012 , 209, 278-281.

(10) Rettenwander, D.; Redhammer, G.; Preishuber-Pflügl, F.; Cheng, L.; Miara, L.; Wagner, R.; Welzl, A.; Suard, E.; Doeff, M. M.; Wilkening, M.; Fleig, J.; Amthauer, G. Structural and Electrochemical Consequences of $\mathrm{Al}$ and $\mathrm{Ga}$ Cosubstitution in $\mathrm{Li}_{7} \mathrm{La}_{3} \mathrm{Zr}_{2} \mathrm{O}_{12}$ Solid Electrolytes. Chem. Mater. 2016, 28, 2384-2392.

(11) Murugan, R.; Thangadurai, V.; Weppner, W. Fast Lithium Ion Conduction in GarnetType $\mathrm{Li}_{7} \mathrm{La}_{3} \mathrm{Zr}_{2} \mathrm{O}_{12}$. Angew. Chemie - Int. Ed. 2007, 46, 7778-7781.

(12) Guin, M.; Tietz, F.; Guillon, O. New Promising NASICON Material as Solid Electrolyte 
for Sodium-Ion Batteries: Correlation between Composition, Crystal Structure and Ionic Conductivity of $\mathrm{Na}_{3+x} \mathrm{Sc}_{2} \mathrm{Si}_{x} \mathrm{P}_{3-x} \mathrm{O}_{12}$. Solid State Ionics 2016, 293, 18-26.

(13) Guin, M.; Tietz, F. Survey of the Transport Properties of Sodium Superionic Conductor Materials for Use in Sodium Batteries. J. Power Sources 2015, 273, 1056-1064.

(14) Arbi, K.; Rojo, J. M.; Sanz, J. Lithium Mobility in Titanium Based Nasicon $\mathrm{Li}_{1+x} \mathrm{Ti}_{2-}$ ${ }_{x} \mathrm{Al}_{x}\left(\mathrm{PO}_{4}\right)_{3}$ and $\mathrm{LiTi}_{2-x} \mathrm{Zr}_{x}\left(\mathrm{PO}_{4}\right)_{3}$ Materials Followed by NMR and Impedance Spectroscopy. J. Eur. Ceram. Soc. 2007, 27, 4215-4218.

(15) El-Shinawi, H.; Greaves, C.; Janek, J. Sol-Gel Synthesis and Room-Temperature Properties of $\alpha-\mathrm{LiZr}_{2}\left(\mathrm{PO}_{4}\right)_{3}$. RSC Adv. 2015, 5, 17054-17059.

(16) Breuer, S.; Prutsch, D.; Ma, Q.; Epp, V.; Preishuber-Pflügl, F.; Tietz, F.; Wilkening, M. Separating Bulk from Grain Boundary Li Ion Conductivity in the Sol-Gel Prepared Solid Electrolyte $\mathrm{Li}_{1.5} \mathrm{Al}_{0.5} \mathrm{Ti}_{1.5}\left(\mathrm{PO}_{4}\right)_{3}$. J. Mater. Chem. A 2015, 3, 21343-21350.

(17) Kamaya, N.; Homma, K.; Yamakawa, Y.; Hirayama, M.; Kanno, R.; Yonemura, M.; Kamiyama, T.; Kato, Y.; Hama, S.; Kawamoto, K.; Mitsui, A. A Lithium Superionic Conductor. Nat. Mater. 2011, 10, 682-686.

(18) Weber, D. A.; Senyshyn, A.; Weldert, K. S.; Wenzel, S.; Zhang, W.; Kaiser, R.; Berendts, S.; Janek, J.; Zeier, W. G. Structural Insights and 3D Diffusion Pathways within the Lithium Superionic Conductor $\mathrm{Li}_{10} \mathrm{GeP}_{2} \mathrm{~S}_{12}$. Chem. Mater. 2016, 28, 59055915.

(19) Kuhn, A.; Gerbig, O.; Zhu, C.; Falkenberg, F.; Maier, J.; Lotsch, B. V. A New Ultrafast Superionic Li-Conductor: Ion Dynamics in $\mathrm{Li}_{11} \mathrm{Si}_{2} \mathrm{PS}_{12}$ and Comparison with Other Tetragonal LGPS-Type Electrolytes. Phys. Chem. Chem. Phys. 2014, 16, 14669-14674.

(20) Krauskopf, T.; Culver, S. P.; Zeier, W. G. Bottleneck of Diffusion and Inductive Effects in $\mathrm{Li}_{10} \mathrm{Ge}_{1-x} \mathrm{Sn}_{x} \mathrm{P}_{2} \mathrm{~S}_{12}$. Chem. Mater. 2018, 30, 1791-1798.

(21) Harm, S.; Hatz, A. K.; Moudrakovski, I.; Eger, R.; Kuhn, A.; Hoch, C.; Lotsch, B. V. Lesson Learned from NMR: Characterization and Ionic Conductivity of LGPS-like Li7 SiPS $_{8}$. Chem. Mater. 2019, 31, 1280-1288.

(22) Dietrich, C.; Weber, D. A.; Sedlmaier, S. J.; Indris, S.; Culver, S. P.; Walter, D.; Janek, J.; Zeier, W. G. Lithium Ion Conductivity in $\mathrm{Li}_{2} \mathrm{~S}-\mathrm{P}_{2} \mathrm{~S}_{5}$ Glasses-Building Units and Local Structure Evolution during the Crystallization of Superionic Conductors $\mathrm{Li}_{3} \mathrm{PS}_{4}, \mathrm{Li}_{7} \mathrm{P}_{3} \mathrm{~S}_{11}$ and $\mathrm{Li}_{4} \mathrm{P}_{2} \mathrm{~S}_{7}$. J. Mater. Chem. A 2017, 5, 18111-18119.

(23) Tatsumisago, M.; Hama, S.; Hayashi, A.; Morimoto, H.; Minami, T. New Lithium Ion Conducting Glass-Ceramics Prepared from Mechanochemical $\mathrm{Li}_{2} \mathrm{~S}-\mathrm{P}_{2} \mathrm{~S}_{5}$ Glasses. Solid State Ionics 2002, 154, 635-640. 
(24) Hayashi, A.; Hama, S.; Morimoto, H.; Tatsumisago, M.; Minami, T. Preparation of Li2 2 $\mathrm{P}_{2} \mathrm{~S}_{5}$ Amorphous Solid Electrolytes by Mechanical Milling. J. Am. Ceram. Soc. 2004, $84,477-479$.

(25) Hayashi, A.; Hama, S.; Minami, T.; Tatsumisago, M. Formation of Superionic Crystals from Mechanically Milled $\mathrm{Li}_{2} \mathrm{~S}-\mathrm{P}_{2} \mathrm{~S}_{5}$ Glasses. Electrochem. commun. 2003, 5, 111-114.

(26) Seino, Y.; Ota, T.; Takada, K.; Hayashi, A.; Tatsumisago, M. A Sulphide Lithium Super Ion Conductor Is Superior to Liquid Ion Conductors for Use in Rechargeable Batteries. Energy Environ. Sci. 2014, 7, 627-631.

(27) Kanno, R.; Murayama, M. Lithium Ionic Conductor Thio-LISICON: The $\mathrm{Li}_{2} \mathrm{~S}-\mathrm{GeS}_{2}-\mathrm{P}$ ${ }_{2} \mathrm{~S}_{5}$ System. J. Electrochem. Soc. 2001, 148, 742-746.

(28) Kanno, R.; Hata, T.; Kawamoto, Y.; Irie, M. Synthesis of a New Lithium Ionic Conductor, Thio-LISICON-Lithium Germanium Sulfide System. Solid State Ionics 2000, 130, 97-104.

(29) Minafra, N.; Culver, S. P.; Li, C.; Senyshyn, A.; Zeier, W. G. Influence of the Lithium Substructure on the Diffusion Pathways and Transport Properties of the Thio-LISICON $\mathrm{Li}_{4} \mathrm{Ge}_{1-x} \mathrm{Sn}_{x} \mathrm{~S}_{4}$. Chem. Mater. 2019, 31, 3794-3802.

(30) Leube, B. T.; Inglis, K. K.; Carrington, E. J.; Sharp, P. M.; Shin, J. F.; Neale, A. R.; Manning, T. D.; Pitcher, M. J.; Hardwick, L. J.; Dyer, M. S.; Blanc, F.; Claridge, J. B.; Rosseinsky, M. J. Lithium Transport in $\mathrm{Li}_{4.4} \mathrm{M}_{0.4} \mathrm{M}^{\prime}{ }_{0.6} \mathrm{~S}_{4}\left(\mathrm{M}=\mathrm{Al}^{3+}, \mathrm{Ga}^{3+}\right.$, and $\mathrm{M}^{\prime}=\mathrm{Ge}^{4+}$, $\mathrm{Sn}^{4+}$ ): Combined Crystallographic, Conductivity, Solid State NMR, and Computational Studies. Chem. Mater. 2018, 30, 7183-7200.

(31) Zhou, L.; Assoud, A.; Shyamsunder, A.; Zhang, Q.; Hartmann, P.; Kulisch, J.; Nazar, L. F. An Entropically Stabilized Fast-Ion Conductor: $\mathrm{Li}_{3.25}\left[\mathrm{Si}_{0.25} \mathrm{P}_{0.75}\right] \mathrm{S}_{4}$, Chem. Mater. 2019. https://doi.org/10.1021/acs.chemmater.9b00657.

(32) Adeli, P.; Bazak, J. D.; Park, K. H.; Kochetkov, I.; Huq, A.; Goward, G. R.; Nazar, L. F. Boosting Solid-State Diffusivity and Conductivity in Lithium Superionic Argyrodites by Halide Substitution. Angew. Chemie - Int. Ed. 2019, 58, 8681-8686.

(33) Deiseroth, H. J.; Kong, S. T.; Eckert, H.; Vannahme, J.; Reiner, C.; Zaiß, T.; Schlosser, M. $\mathrm{Li}_{6} \mathrm{PS}_{5} \mathrm{X}$ : A Class of Crystalline Li-Rich Solids with an Unusually High $\mathrm{Li}^{+}$Mobility. Angew. Chemie - Int. Ed. 2008, 47, 755-758.

(34) Rayavarapu, P. R.; Sharma, N.; Peterson, V. K.; Adams, S. Variation in Structure and Li ${ }^{+}$-Ion Migration in Argyrodite-Type $\mathrm{Li}_{6} \mathrm{PS}_{5} \mathrm{X}(\mathrm{X}=\mathrm{Cl}$, Br, I) Solid Electrolytes. J. Solid State Electrochem. 2012, 16, 1807-1813.

(35) Hanghofer, I.; Brinek, M.; Eisbacher, S. L.; Bitschnau, B.; Volck, M.; Hennige, V.; 
Hanzu, I.; Rettenwander, D.; Wilkening, H. M. R. Substitutional Disorder: Structure and Ion Dynamics of the Argyrodites $\mathrm{Li}_{6} \mathrm{PS}_{5} \mathrm{Cl}, \mathrm{Li}_{6} \mathrm{PS}_{5} \mathrm{Br}$ and $\mathrm{Li}_{6} \mathrm{PS}_{5} \mathrm{I}$. Phys. Chem. Chem. Phys. 2019, 21, 8489-8507.

(36) Ohno, S.; Helm, B.; Fuchs, T.; Dewald, G.; Kraft, M. A.; Culver, S. P.; Senyshyn, A.; Zeier, W. G. Further Evidence for Energy Landscape Flattening in the Superionic Argyrodites $\mathrm{Li}_{6+x} \mathrm{P}_{1-x} \mathrm{M}_{x} \mathrm{~S}_{5} \mathrm{I}(\mathrm{M}=\mathrm{Si}, \mathrm{Ge}, \mathrm{Sn})$. Chem. Mater. 2019, 31, 4936-4944.

(37) Kraft, M. A.; Ohno, S.; Zinkevich, T.; Koerver, R.; Culver, S. P.; Fuchs, T.; Senyshyn, A.; Indris, S.; Morgan, B. J.; Zeier, W. G. Inducing High Ionic Conductivity in the Lithium Superionic Argyrodites $\mathrm{Li}_{6+x} \mathrm{P}_{1-x} \mathrm{Ge}_{x} \mathrm{~S}_{5} \mathrm{I}$ for All-Solid-State Batteries. J. Am. Chem. Soc. 2018, 140, 16330-16339.

(38) Kraft, M. A.; Culver, S. P.; Calderon, M.; Böcher, F.; Krauskopf, T.; Senyshyn, A.; Dietrich, C.; Zevalkink, A.; Janek, J.; Zeier, W. G. Influence of Lattice Polarizability on the Ionic Conductivity in the Lithium Superionic Argyrodites $\mathrm{Li}_{6} \mathrm{PS}_{5} \mathrm{X}(\mathrm{X}=\mathrm{Cl}, \mathrm{Br}, \mathrm{I}) . J$. Am. Chem. Soc. 2017, 139, 10909-10918.

(39) Minafra, N.; Culver, S. P.; Krauskopf, T.; Senyshyn, A.; Zeier, W. G. Effect of Si Substitution on the Structural and Transport Properties of Superionic Li-Argyrodites. $J$. Mater. Chem. A 2018, 6, 645-651.

(40) Yubuchi, S.; Uematsu, M.; Deguchi, M.; Hayashi, A.; Tatsumisago, M. Lithium-IonConducting Argyrodite-Type $\mathrm{Li}_{6} \mathrm{PS}_{5} \mathrm{X}(\mathrm{X}=\mathrm{Cl}, \mathrm{Br}, \mathrm{I})$ Solid Electrolytes Prepared by a Liquid-Phase Technique Using Ethanol as a Solvent. ACS Appl. Energy Mater. 2018, 1, $3622-3629$.

(41) Rao, R. P.; Adams, S. Studies of Lithium Argyrodite Solid Electrolytes for All-SolidState Batteries. Physica Status Solidi (A) Applications and Materials Science. 2011, pp 1804-1807.

(42) Chen, M.; Adams, S. High Performance All-Solid-State Lithium/Sulfur Batteries Using Lithium Argyrodite Electrolyte. J. Solid State Electrochem. 2015, 19, 697-702.

(43) Koerver, R.; Aygün, I.; Leichtweiß, T.; Dietrich, C.; Zhang, W.; Binder, J. O.; Hartmann, P.; Zeier, W. G.; Janek, J. Capacity Fade in Solid-State Batteries: Interphase Formation and Chemomechanical Processes in Nickel-Rich Layered Oxide Cathodes and Lithium Thiophosphate Solid Electrolytes. Chem. Mater. 2017, 29, 5574-5582.

(44) Sakuda, A.; Hayashi, A.; Tatsumisago, M. Sulfide Solid Electrolyte with Favorable Mechanical Property for All-Solid-State Lithium Battery. Sci. Rep. 2013, 3, 2261.

(45) De Klerk, N. J. J.; Rosłoń, I.; Wagemaker, M. Diffusion Mechanism of Li Argyrodite Solid Electrolytes for Li-Ion Batteries and Prediction of Optimized Halogen Doping: The 
Effect of Li Vacancies, Halogens, and Halogen Disorder. Chem. Mater. 2016, 28, 79557963.

(46) Bernges, T.; Culver, S. P.; Minafra, N.; Koerver, R.; Zeier, W. G. Competing Structural Influences in the Li Superionic Conducting Argyrodites $\mathrm{Li}_{6} \mathrm{PS}_{5-x} \mathrm{Se} e_{x} \mathrm{Br}(0 \leq x \leq 1)$ upon Se Substitution. Inorg. Chem. 2018, 57, 13920-13928.

(47) Kong, S. T.; Gün, Ö.; Koch, B.; Deiseroth, H. J.; Eckert, H.; Reiner, C. Structural Characterisation of the Li Argyrodites $\mathrm{Li}_{7} \mathrm{PS}_{6}$ and $\mathrm{Li}_{7} \mathrm{PSe}_{6}$ and Their Solid Solutions: Quantification of Site Preferences by MAS-NMR Spectroscopy. Chem. - A Eur. J. 2010, $16,5138-5147$.

(48) Wang, Y.; Richards, W. D.; Ong, S. P.; Miara, L. J.; Kim, J. C.; Mo, Y.; Ceder, G. Design Principles for Solid-State Lithium Superionic Conductors. Nat. Mater. 2015, 14, 10261031.

(49) Krauskopf, T.; Pompe, C.; Kraft, M. A.; Zeier, W. G. Influence of Lattice Dynamics on $\mathrm{Na}^{+}$Transport in the Solid Electrolyte $\mathrm{Na}_{3} \mathrm{PS}_{4-x} \mathrm{Se}_{x}$. Chem. Mater. 2017, 29, 8859-8869.

(50) Krauskopf, T.; Muy, S.; Culver, S. P.; Ohno, S.; Delaire, O.; Shao-Horn, Y.; Zeier, W. G. Comparing the Descriptors for Investigating the Influence of Lattice Dynamics on Ionic Transport Using the Superionic Conductor $\mathrm{Na}_{3} \mathrm{PS}_{4-x} \mathrm{Se}_{x} . J$. Am. Chem. Soc. 2018, 140, 14464-14473.

(51) Muy, S.; Bachman, J. C.; Giordano, L.; Chang, H. H.; Abernathy, D. L.; Bansal, D.; Delaire, O.; Hori, S.; Kanno, R.; Maglia, F.; Lupart, S.; Lamp, P.; Shao-Horn, Y. Tuning Mobility and Stability of Lithium Ion Conductors Based on Lattice Dynamics. Energy Environ. Sci. 2018, 11, 850-859.

(52) Rice, M. J.; Roth, W. L. Ionic Transport in Super Ionic Conductors: A Theoretical Model. J. Solid State Chem. 1972, 4, 294-310.

(53) Vineyard, G. H. Frequency Factors and Isotope Effects in Solid State Rate Processes. J. Phys. Chem. Solids 1957, 3, 121-127.

(54) Brüesch, P.; Pietronero, L.; Strässler, S.; Zeller, H. R. Brownian Motion in a Polarizable Lattice: Application to Superionic Conductors. Phys. Rev. B 1977, 15, 4631-4637.

(55) Hanghofer, I.; Gadermaier, B.; Wilkening, H. M. R. Fast Rotational Dynamics in Argyrodite-Type $\mathrm{Li}_{6} \mathrm{PS}_{5} \mathrm{X}(\mathrm{X}: \mathrm{Cl}, \mathrm{Br}, \mathrm{I})$ as Seen by 31P Nuclear Magnetic Relaxation On Cation-Anion Coupled Transport in Thiophosphates. Chem. Mater. 2019, 31, 45914597.

(56) Coelho, A. A. TOPAS and TOPAS-Academic: An Optimization Program Integrating Computer Algebra and Crystallographic Objects Written in C++: An. J. Appl. 
Crystallogr. 2018, 51, 210-218.

(57) Shannon, R. D. Revised Effective Ionic Radii and Systematic Studies of Interatomie Distances in Halides and Chaleogenides Bond Strength-Bond Length Relationships , and Plots of ( 1 ) Radii vs Volume, ( 2 ) Radii vs Coordination Partial Occupancy of Cation Sites , Coval. Acta Crystallogr. 1976, A32, 751.

(58) Denton, A. R.; Ashcroft, N. W. Vegards Law. Phys. Rev. A 1991, 43, 3161-3164.

(59) Epp, V.; Gün, Ö.; Deiseroth, H. J.; Wilkening, M. Long-Range Li+ Dynamics in the Lithium Argyrodite $\mathrm{Li}_{7} \mathrm{PSe}_{6}$ as Probed by Rotating-Frame Spin-Lattice Relaxation NMR. Phys. Chem. Chem. Phys. 2013, 15, 7123-7132.

(60) Sagotra, A. K.; Chu, D.; Cazorla, C. Influence of Lattice Dynamics on Lithium-Ion Conductivity: A First-Principles Study. Phys. Rev. Mater. 2019, 3, 035405.

(61) Rao, M. N.; Goel, P.; Choudhury, N.; Chaplot, S. L., Subrata, G. Lattice Dynamics and Inelastic Neutron Scattering Experiments on andalusite, $\mathrm{Al}_{2} \mathrm{SiO}_{5}$.Solid State Comm. 2002, 121, 333-338.

(62) Kolev, N.; Bontchev, R. P.; Jacobson, A. J.; Popov, V. N.; Hadjiev, V. G.; Litvinchuk, A. P.; Iliev, M. N. Raman Spectroscopy of $\mathrm{CaCu}_{3} \mathrm{Ti}_{4} \mathrm{O}_{12}$. Phys. Rev. B, 2012, 66, 132102.

(63) Stirling, W. G. Neutron Inelastic Scattering Study of the Lattice Dynamics of Strontium Titanate: Harmonic Models. J. Phys. C Solid State Phys. 1972, 5, 2711-2730.

(64) Sturhahn, W.; Toellner, T.S.; Alp, E. E.; Zhang, X.; Ando, M.; Yoda, Y.; Kikuta, S.; Seto, M.; Kimball, C. W.; Dabrowski, B. Phonon Density of States Measured by Inelastic Nuclear Resonant Scattering. Phys. Rev.1958, 74, 326-328.

(65) Agne, M. T.; Hanus, R.; Snyder, G. J. Minimum Thermal Conductivity in the Context of: Diffuson -Mediated Thermal Transport. Energy Environ. Sci. 2018, 11, 609-616.

(66) West, A.; Irvine, J.; Sinclair, D. Electroceramics : Characterization by Impedance Spectroscopy. Adv. Mater. 1990, 2, 132-138.

(67) Weiss, M.; Weber, D. A.; Senyshyn, A.; Janek, J.; Zeier, W. G. Correlating Transport and Structural Properties in $\mathrm{Li}_{1+x} \mathrm{Al}_{x} \mathrm{Ge}_{2-x}\left(\mathrm{PO}_{4}\right)_{3}$ (LAGP) Prepared from Aqueous Solution. ACS Appl. Mater. Interfaces 2018, 10, 10935-10944.

(68) Mariappan, C. R.; Yada, C.; Rosciano, F.; Roling, B. Correlation between MicroStructural Properties and Ionic Conductivity of $\mathrm{Li}_{1.5} \mathrm{Al}_{0.5} \mathrm{Ge}_{1.5}\left(\mathrm{PO}_{4}\right)_{3}$ Ceramics. J. Power Sources 2011, 196, 6456-6464.

(69) Brug, G. J.; van den Eeden, A. L. G.; Sluyters-Rehbach, M.; Sluyters, J. H. The Analysis of Electrode Impedances Complicated by the Presence of a Constant Phase Element. $J$. Electroanal. Chem. 1984, 176, 275-295. 
For table of contents only

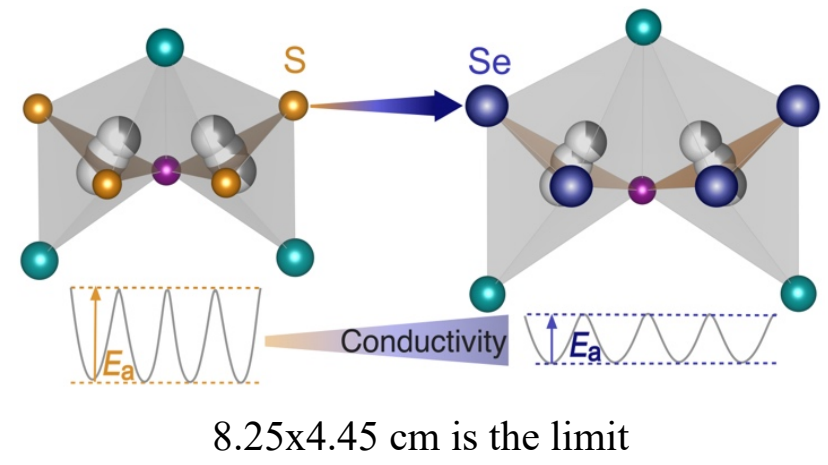

